\title{
Development of a four-channel cardiovascular signals collector based on STM32 with digital-controlled gain
}

\author{
Longcong Chen ${ }^{1}$, Ruiying Zhang ${ }^{1}$, Gaiqin Liu $^{2 *}$, Bin Gao ${ }^{1}$, Guohu Hu${ }^{1}$ \\ ${ }^{1}$ Laboratory of Forensic Medicine and Biomedical Information, Chongqing Medical University, Chongqing, China \\ ${ }^{2}$ School of Opto-Electronic Information, Chongqing University of Technology, Chongqing, China \\ Email: ${ }^{*} \operatorname{lgq}$. abcd@163.com
}

Received 19 February 2013; revised 20 March 2013; accepted 27 March 2013

Copyright (C) 2013 Longcong Chen et al. This is an open access article distributed under the Creative Commons Attribution License, which permits unrestricted use, distribution, and reproduction in any medium, provided the original work is properly cited.

\begin{abstract}
To get better methods on diagnosis of cardiovascular diseases and health assessment by comparison analysis of four kinds of cardiovascular signals, which are blood pressure signal (BPS), plus wave signal (PWS), electrocardiosignal (ECS) and cardiac sound signal (CSS), a four-channel signals collector is introduced in this article. This collector is composed of microcomputer STM32F103RB with integrated 12bit analog to digital converters (ADCs) and some integrated chips (IC) such as PGA113, a programmable gain amplifier with internal calibration channels for system-level calibration. Therefore, the data of four kinds of cardiovascular signals can be both collected by this signal collector with a high precision and output to a computer by universal serial bus (USB) interface, which can also offers power for this instrument. In addition, the gain of input signal can be changed by computer software through USB interface under microchip commands. Thus it has many merits, such as the small volume, low cost, high performance and precision, digital-controlled gain and so on. Consequently, it is convenient to acquire the data of four kinds of cardiovascular signals by it, and a waveform of one sample is shown.
\end{abstract}

Keywords: Cardiovascular Signal; Signal Collector; Digital-Controlled; STM32F103RB; PGA113

\section{INTRODUCTION}

Cardiovascular disease (CD) is a serious disease that is harmful to human health, and it is the main reason to cause death. In recent years, the incidence of $C D$ is increasing, and $\mathrm{CD}$ is regarded as one of the three major diseases that are harmful to human health by World

${ }^{*}$ Corresponding author.
Health Organization. Therefore, Studying on the basic laws of blood flow in human body has important practical and clinical significance. But, the type of CD is various, and its' causes is complex. Among them, many dynamic factors of blood circulation process, such as blood flow, the deformation of blood cells and blood vessels, interaction relationship, play a major role in the disease. Specially, arterial blood flow is more important in cardiovascular system and among $\mathrm{CD}$ such as coronary heart disease, atherosclerosis, the formation of the development process and treatment. The obstacles of arterial blood can cause cardiovascular system physical abnormalities and even result in serious danger. Research shows that blood flow dynamics factors, such as vascular wall shear stress and elasticity, are closely related to atherosclerosis and aneurysm. Thence, the dynamic information of vascular wall [1-4], including variation of BPS, PWS, ECS and CSS, is closely linked to healthy status, especially to disease about heart and blood. Take BPS as an example, BPS is a powerful, consistent and essential risk factor for the diagnosis of cardiovascular diseases [5]. Clinically, it is also one of the most crucial signals for health assessment. However, the measurement of those four kinds of cardiovascular signals still lacks accuracy and is carried out independently in general. As well known, those four kinds of cardiovascular signals are close relationship each other. And more information about cardiovascular diseases can be gained by analyzing their relationships. Therefore, it is important and necessary to develop a collector that may synchronously acquire BPS, PWS, ECS and CSS with high precision for getting better methods on diagnosis of cardiovascular diseases and health assessment.

\section{INSTRUMENT STRUCTION}

Design of the collector includes two sides, hardware and software. Hardware is divided into five units: microcon- 
troller, preamplifiers, filter, programmable gain amplifier and power source unit, and its block diagram is shown in Figure 1.

This collector is mainly based on microcontroller and high-performance integrated chips, such as STM32F103 RB, PGA113, AD620, AD8639, which can increase its intelligence, precision, stability and reduce its power consumption and volume. Four kinds of cardiovascular signals, BPS, PWS, ECS and CSS, can be input into it. Those signals respectively go through preamplifiers, filters and programmable gain amplifiers in order. And then, in order to convert analog cardiovascular signals into corresponding digital data, they are input into two 12-bit ADCs integrated in STM32F103RB. Controlled by microcontroller, four pieces of PGA113 may respecttively produce right gain and enough amplitude for ADCs. The data of four kinds of cardiovascular signals, acquired by $\mathrm{ADC}$ and direct memory access (DMA) integrated in STM32F103RB, may be output to a computer by USB interface, which can also supply power for this collector. In addition, the gain of input signal can be changed by computer software through USB interface under microchip control. As a result, it has many merits, such as the small volume, low cost, high performance and precision, digital-controlled gain and so on.

The software of this collector was designed by Kiel uVision3 for STM32F103RB.

The following, we mainly describe this collector's four units of hardware, which are microcontroller, preamplifiers, filters, programmable gain amplifiers, and software in details.

\subsection{The Main Hardware}

The important and vital units in this collector are microcontroller unit, preamplifier unit, filter unit and programmable gain amplifier unit. Therefore, we only describe those four units in details.

\subsubsection{Mircocontroller Unit}

The microcontroller unit includes microprocessor STM-

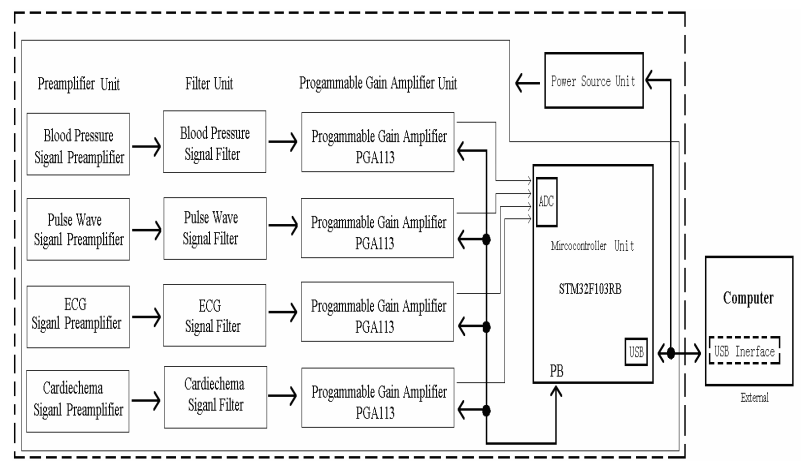

Figure 1. Block diagram of collector.
32F103RB [6] and its peripheral components. STM32 F103RB, a single-chip microcontroller, based on a highperformance ARM 32-bit Cortex ${ }^{\mathrm{TM}}-\mathrm{M} 3 \mathrm{CPU}$, can work in $72 \mathrm{MHz}$ maximum frequency, has USB $2.012 \mathrm{Mbs}$ full speed, 7-channel DMA controller, $2 \times 12$-bit and $1 \mu \mathrm{s} \mathrm{A} /$ $\mathrm{D}$ converters whose conversion range is 0 to $3.6 \mathrm{~V}$, with $128 \mathrm{~K}$ bytes Flash program memory, $20 \mathrm{~K}$ bytes SRAM memory, a Watchdog Timer. Furthermore, two 12-bit onchip ADCs are used to measure four cardiovascular signals. At the same time, STM32F103RB outputs digital data to control the gain of PGA113 according to instructions delivered by computer through USB interface, by which microcontroller can transmit signals data to computer. In a word, STM32F103RB plays a major role in controlling other parts to realize data exchange and corresponding function in this collector.

\subsubsection{Preamplifiers Unit}

The basic aim of this unit is to get BPS, PWS, ECS and CSS with lowest noise. This unit mainly includes four preamplifiers: BPS preamplifier, PWS preamplifier, ECS preamplifier and CSS preamplifier. They are composed of ICs such as AD620, OP2177 and its peripheral components. AD620 works well as a preamplifier due to its low input voltage noise of $9 \mathrm{nV} / \sqrt{\mathrm{Hz}}$ at $1 \mathrm{kHz}, 0.28$ $\mu \mathrm{Vp}-\mathrm{p}$ in the $0.1 \mathrm{~Hz}$ to $10 \mathrm{~Hz}$ band, $0.1 \mathrm{pA} / \sqrt{\mathrm{Hz}}$ input current noises and the low input bias current of $1.0 \mathrm{nA}$ max. And it is not only a both three op-amp differential amplifier, which has high anti-interference, but also a high accuracy instrumentation amplifier that requires only one external resistor to set gains of 1 to 10,000 . Besides, AD620 has some outstanding features such as high accuracy of $40 \mathrm{ppm}$ maximum nonlinearity, low offset voltage of $50 \mu \mathrm{V}$ max, offset drift of $0.6 \mu \mathrm{V} /{ }^{\circ} \mathrm{C}$ max. Furthermore, the low noise, low input bias current and low power of the AD620 make it well suited for our ECG and noninvasive blood pressure measure. In this unit, every channel uses AD620 as preamplifier, especially for ECS one. OP2177 is added in order to drive right leg and maintain stability of the right leg drive loop, because OP2177, operational amplifier, has typical offset voltage of $15 \mu \mathrm{V}$, extremely small temperature drift of $0.2 \mu \mathrm{V} /{ }^{\circ} \mathrm{C}$, very low input bias current of $2 \mathrm{nA}$ maximum, gain bandwidth of $1.3 \mathrm{MHz}$. So it is an ideal selection for the right leg drive loop. After processing of this unit, the enough amplitude signals can be acquired for next filter one.

\subsubsection{Filter Unit}

The main purpose of this unit, which is composed of AD8639, and its peripheral components, is to extract useful signal from BPS, PWS, ECS and CSS, and restrain interference one. Because different signal have different frequency features, this four-channel signals have 
their own corresponding active second-order filters. For $\mathrm{BPS}$, an active second-order and $\mathrm{RC}$ low pass filter, whose cutoff frequency is $1000 \mathrm{~Hz}$, is designed, and for PWS, a band pass filter of frequency between $0.3 \mathrm{~Hz}$ and $40 \mathrm{~Hz}$. For ECS, a bandpass filter of frequency between 0.05 and $120 \mathrm{~Hz}$, and a notch filter of frequency $50 \mathrm{~Hz}$, are must be added in its signal channel. For CSS, a bandpass filter of frequency between 0.05 and $2 \mathrm{KHz}$, is applied. All above filters are realized by using AD8639, because it has typical offset voltage of only $3 \mu \mathrm{V}$, extremely small temperature drift of $0.01 \mu \mathrm{V} /{ }^{\circ} \mathrm{C}$, very low input bias current of $40 \mathrm{pA}$ maximum, gain bandwidth of 1.5 MHz. After processing of those filters, every channel signal has its own needed frequency bandwidth and is input into next unit.

\subsubsection{Progammable Gain Amplifier Unit}

The primary goal of this unit is to get a suitable digitalcontrolled gain for input signal and to avoid that signal amplitude is too big or too small for ADCs integrated in microcontroller. Therefore, we selected four piece of PAG113 [7], programmable gain amplifier, to get different amplification factors for every channel and suit for different persons. PGA113 is single-ended input, singlesupply, programmable gain amplifier (PGA) with an input multiplexer (MUX). The multiplexer channel selection and gain selection may be done through a standard serial peripheral interface (SPI). PAG113, whose block diagram is shown in Figure 2, has a two-channel input MUX and provide scope gain selections (1, 2, 5, 10, 20, 50, 100, and 200). All models use split-supply architecture with an analog supply, $\mathrm{AV}_{\mathrm{DD}}$, and a digital supply, $\mathrm{DV}_{\mathrm{DD}}$. This split-supply architecture allows for ease of interface to analog-to-digital converters (ADCs), microcontrollers in mixed-supply voltage systems, where the analog supply is $+5 \mathrm{~V}$ and the digital supply are $+3 \mathrm{~V}$, is applied in this collector and shown in Figure 2. Especially, four internal calibration channels are provided for system-level calibration and can improve precision of

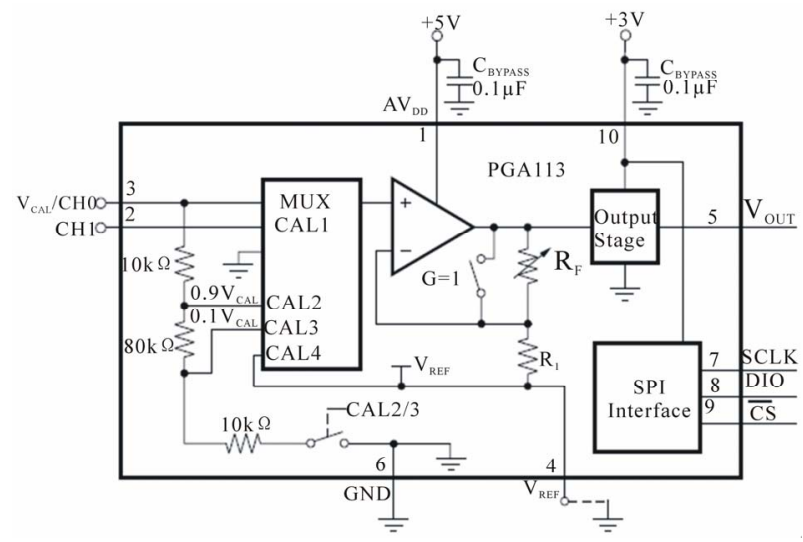

Figure 2. Function block diagram of PGA113. the collector. The channels of PGA113 are tied to GND, $0.9 \mathrm{~V}_{\mathrm{CAL}}, 0.1 \mathrm{~V}_{\mathrm{CAL}}$, and $\mathrm{V}_{\mathrm{REF}}$, respectively. In this unit, $\mathrm{V}_{\mathrm{CAL}}$, an external high-precision voltage of $3.0 \mathrm{~V}$, which is connected to pin $2, \mathrm{~V}_{\mathrm{CAL}} / \mathrm{CH} 0$, acts as the system calibration and $\mathrm{ADC}$ reference. As a result, the precision of measuring signals can be improved markedly. In addition, PGA113 also offers a software-controlled shutdown feature for low standby power and has a three-wire SPI digital interface.

Therefore, after STM32F103RB sends corresponding data to PGA113 by SPI, gain and offset calibration of ADC and input signals can be obtained. Above all, controlled by STM32F103RB, every channel signal can be output from this unit with enough amplitude and input into 12-bit and $1 \mu \mathrm{s}$ ADCs integrated in STM32F103RB.

\subsection{Design of Software}

To realize the function, the corresponding software of this collector must be designed, and we completed it by Kiel uVision3 for STM32F103RB with C language. The software, whose control flow is shown in Figure 3, is primarily made up of one main program and four subprograms, which are signal gain regulation control subprogram, ADC control subprogram, USB control subprogram and calibration subprogram. The main program calls USB control subprogram to obtain the input information, such as calibration, gain and sample frequency of every channel signal, and then the signal gain regulation control subroutine send command to PGA113 for obtaining needed gain about corresponding channel. At the same time, main program calls ADC control subprogram to realize their sample frequency for every kind of signal. Certainly, main program of collector would call corresponding calibration subprogram to control PGA113 select channel $\mathrm{V}_{\mathrm{CAL}} / \mathrm{CHO}$ and realize calibration if user send calibration command to collector by computer software.

Signal gain regulation control subprogram manages

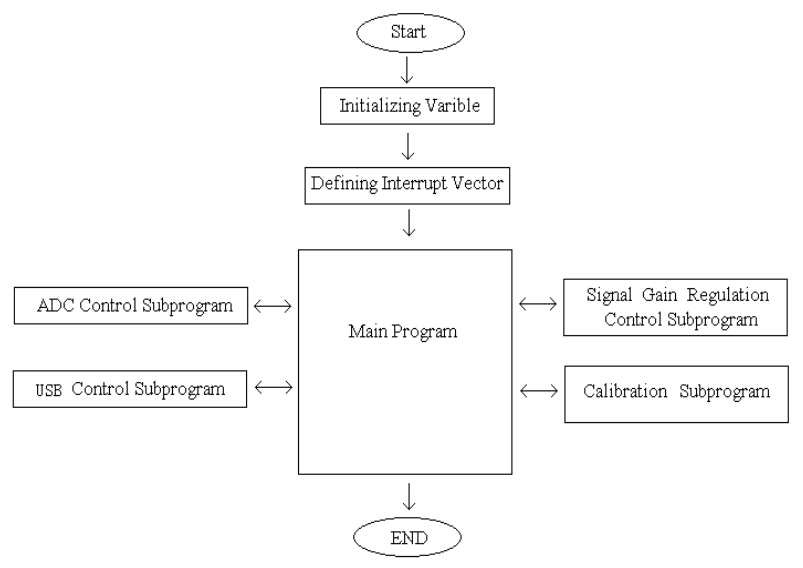

Figure 3. Software control flow. 
STM32F103RB send the command to PGA113 by SPI in order to select corresponding channel of PGA113 and gain for every signal. By setting timers and DMA of microchip, ADC control subprogram to realize the function that changing analog signal to 12-bit digital data with given sample frequency and saving those data to defined buffer. USB control subprogram realizes exchange of information and data with computer by USB interface. Calibration subprogram is for correcting data of every channel by making PGA113 select channel $\mathrm{V}_{\mathrm{CAL}} / \mathrm{CH} 0$. By this software of this instrument, information exchange can be completed with computer, and the waveform of corresponding signals may be displayed by computer software.

\section{DISUSSIONS AND CLOUSIONS}

This collector adopts 32-bit Cortex ${ }^{\mathrm{TM}}-\mathrm{M} 3$ CPU STM 32F103RB and high-performance chips, such as AD620 and PGA113. As a result, the data of four kinds of cardiovascular signals can be collected with a high precision for using PGA113 and an external high-precision voltage reference, and may be output to computer by USB, which can also offers power for this collector. In addition, the gain of input signal can be modified by computer software through USB interface under microchip command.

For testing performance of this collector, we get an example of four signals, whose waveforms are shown in Figure 4. Among the four waveforms, A shows waveform of BPS when the pressure of gas band was slowly changing from large to low by none-invasive blood pressure measure, and B shows waveform of PWS under above same condition. C and D show waveform of ECSand CSS respectively. From Figure 4, some relationships of four cardiovascular signals could be gained. Consequently, by using in practice, it not only is convenient

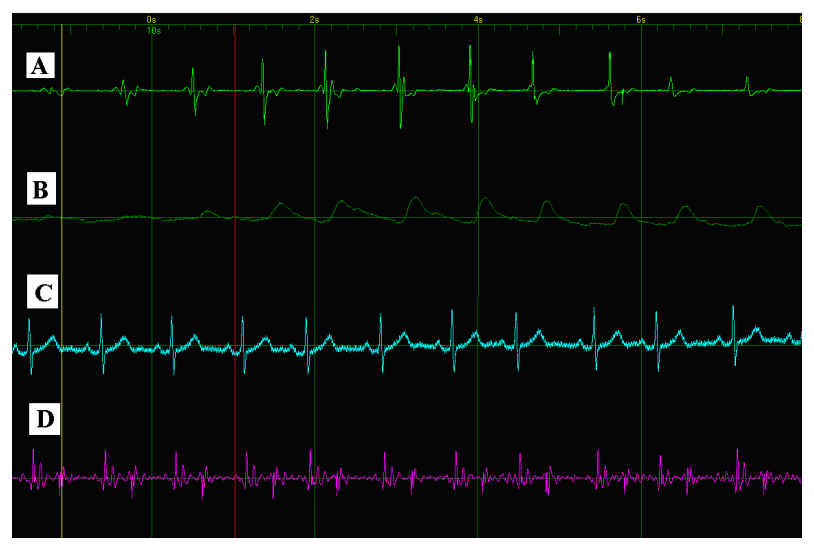

Figure 4. A example for application of this collector. (A) shows BPS when extern pressure changed, (B) shows PWS when extern pressure changed, (C) shows ECS, (D)shows CSS. to be operated, but also has extremely high working stability and reliability.

Therefore, we could confidently draw such a conclusion that this collector is very convenient to get needed four kinds of cardiovascular signals. And it has many merits, such as the small volume, low cost, high performance and precision, digital-controlled gain and so on. In addition, it can be widely used in studying on BPS, PWS, ECS, CSS and their relationships.

\section{REFERENCES}

[1] Andrew, M. (2006) Wilson are vascular function measurement ready for the clinic. European Heart Journal, 27, 255-257.

[2] Lamarre-Cliché, M., Cheong, N.N.G. and Larochelle, P. (2011) Comparative assessment of four blood pressure Measurement methods in hypertensives. Canadian Journal of Cardiology, 27, 455-460. doi:10.1016/j.cjca.2011.05.001

[3] Romagnoli, S., Romano, S.M., Bevilacqua, S., et al. (2011) Dynamic response of liquid-filled catheter systems for measurement of blood pressure: Precision of measurements and reliability of the Pressure Recording Analytical Method with different disposable systems. Journal of Critical Care, 26, 415-422.

doi:10.1016/j.jcrc.2010.08.010

[4] Lan, H., Al-Jumaily, A.M., Loweb, A. and Hing, W. (2011) Effect of tissue mechanical properties on cuff-based blood pressure measurements. Medical Engineering \& Physics, 33, 1287-1292. doi:10.1016/j.medengphy.2011.06.006

[5] Pickering, T.G., et al. (2005) Recommendations for blood pressure measurement in humans and experimental animals: Part 1: Blood pressure measurement in humans: A statement for professionals from the Subcommittee of Professional and Public Education of the American Heart Association Council on high blood pressure research. $\mathrm{Hy}$ pertension, 45, 142-161.

[6] STMicroelectronics (2013) Medium-density performance line ARM-based 32-bit MCU with 64 or $128 \mathrm{~KB}$ Flash, USB, CAN, 7 timers, 2 ADCs, 9 com Interfaces.

http://www.st.com/st-web-ui/static/active/en/resource/tec hnical/document/datasheet/CD00161566.pdf

[7] Texas Instruments (2013) Zerø-drift programmable gain amplifier with mux. http://www.ti.com/lit/ds/symlink/pga113.pdf 